\title{
Direct and indirect methods of evaluating the NETosis process
}

\author{
Weronika Kasprzycka', Iwona Homa-Mlak', Radosław Mlak', Teresa Małecka-Massalska' \\ ${ }^{1}$ Chair and Department of Human Physiology, Medical University, Lublini, Poland
}

Kasprzycka W, Homa-Mlak I, Mlak R, Małecka-Massalska T. Direct and indirect methods of evaluating the NETosis process. J Pre-Clin Clin Res. 2019; 13(1): 50-56. doi: 10.26444/jpccr/105563

\begin{abstract}
In response to various stimuli, neutrophils may release extracellular network (NET - neutrophil extracellular trap) consisting of DNA, proteolytic enzymes and other components of the cell nucleus. The NETosis process was first described in 2004 by Brinkmann et al. as animmunological response to Gram-positive bacteria, Gram-negative bacteria. Other sources provide data referring to the created network in response to the activity of fungi, protozoa and viruses. It is a mechanism of programmed cell death that leads to chromatin decondensation in the nucleus, disintegration of cell organelles and mixing of their constituent, as well as cell membrane permeabilization. The ability to release similar networks is also demonstrated by mast cells (MCET - mast cell extracellular trap), eosinophils (EET - eosinophil extracellular trap) and macrophages (MET macrophage extracellular trap). Various microscopy techniques, for example, immunofluorescence microscopy, transmission electron microscopy, and scanning electron microscopy, flow cytometry and ELISA tests are used to better illustrate and evaluate the NETosis markers. Current knowledge regarding the formation of NETs suggests in-vitro qualitative microscopic examination. So far, measurements based on flow cytometry allow for quick and objective evaluation of several thousand cells simultaneously. The application of cytometry facilitates indirect detection of NET producing cells in blood samples. While ELISA technique, due to the simplicity of making measurements and wide availability of validated tests, may contribute to its routine usage as a tool in screening tests.
\end{abstract}

\section{Key words}

neutrophils, NET, flow cytometry, electron microscopy, ELISA.

\section{INTRODUCTION}

Neutrophils (PMN - polymorphonuclear cells) have developed many ways to destroy pathogens, such as phagocytosis, NETs (neutrophil extracellular traps). In response to inflammatory stimuli, neutrophils can migrate from circulating blood to the infected tissues, where they effectively absorb and inactivate bacteria. Bacteria are quickly killed by proteolytic enzymes and antibacterial proteins [1]. In 2004, Brinkmann et al. were the first to present a not previously described process responsible for the destruction of micro-organisms - by means of NETs composed of DNA, granule components (including proteolytic enzymes) and the contents of the cell nucleus, which are released into the extracellular space [2, $3,4]$. This network is formed in response to the activity of Gram-positive and Gram-negative bacteria $[2,5,6,7]$. Other sources provide some data referring to the created network, in response to the activity of fungi $[8,9,10]$, protozoa $[11$, 12 ] and viruses [12]. The pathogens trapped in the chromatin NET are subjected to the action of various substances, such as histone proteins (core and $\mathrm{H} 1$ linker histones), cationic serine proteases (proteinase 3, cathepsin G, NE - neutrophil elastase), MPO (myeloperoxidase), BPI protein (bactericidal/ permeability-increasing protein with bactericidal properties increasing permeability), lactoferrin, gelatinase B, cathelicidin (LL-37 or CAP-18 (cathelicidin antimicrobial peptide) and tryptase [13]. Table 1 below presents NET components that are significant in the process of NETosis.

Address for correspondence: Weronika Kasprzycka, Chair and Department of Human Physiology, Medical University, Lublini, Poland

E-mail: weronikakasprzycka2@gmail.com

Received: 2 January 2019; accepted: 12 March 2019; first published: 21 March 2019
Inducing factors, components and processes similar to NETosis. After in vitro activation with PMA (phorbol myristate acetate), LPS (lipopolysaccharide), activated blood platelets, cytokines: interleukin 8 (IL-8), tumour necrosis factor $-\alpha($ TNF $-\alpha)$, NET is formed. These substances, when used individually, e.g. PMA or PMA in combination with IL-8, may trigger NETosis. Additionally, this process may also be observed at sites of inflammation, e.g. in patients with small vessel vasculitis, SLE (systemic lupus erythematosus), malaria (associated with the formation of anti-neutrophil antibodies), in streptococcal infection or appendicitis [14, $15,16,17]$, as well as in patients with sepsis (associated with increased thrombocyte activation by LPS mediated by TLR4 (toll-like receptors). Platelets activated in this way bind to immobilised endothelium-bound neutrophils, and in this way, in just 5-10 minutes they stimulate them to form NETs $[18,19]$. Depending on the factor that activated the formation and release of NET, its formation may take from a few minutes to several hours, e.g. after stimulation with PMA, NET release takes place within 1-4 hours. [17, 20]. For NET release, the formation of ROS (reactive oxygen species) is also required. The stimulated neutrophils, e.g. PMA, activate NADPH oxidase, which leads to the formation of a huge number of peroxides and NET release [21].

Thanks to the use of very sensitive methods (e.g. electron microscopy), it is possible to precisely determine the composition of the NET released by neutrophils to the extracellular environment. It consists of chromatin fibres, 15-17 $\mathrm{nm}$ in diameter, which contain DNA and histones (H1, H2A, H2B, H3 and H4). The structure of DNA fibres is maintained thanks to many proteins (MPO, calprotectin, MMP-9, BPI), which indirectly or directly affect the death of bacteria, fungi and protozoa [17]. 
Eosinophilic granulocytes also participate in the release of EET (eosinophil extracellular traps). As a result of stimulation of these cells by, e.g. LPS or complement component 5a (C5a), the mitochondrial DNA is ejected from the cell along with ECP (eosinophil cationic protein) and MBP (major basic protein) [22]. It should be noted, however, that the network released by eosinophils is significantly different from the NET and MCET. Chromatin comes from mitochondria, therefore it does not contain histone proteins in its structure. Additionally, the nuclear envelope of eosinophils is intact, resulting in the process of EET formation not being directly related to cell death [22]. Establishment of the EET has been found in some cases of skin diseases, such as the creeping myiasis syndrome and Crohn's disease [23].

Macrophages have the ability to release chromatin extracellular networks. In 2010, Chow et al. [24] described macrophage subpopulation in mice - (used to develop RAW 264.7 cell line), which after stimulation with PMA were able to form extracellular networks which the authors named 'acrophage extracellular traps'(MET). Their release, as the case of neutrophils and mastocytes, leads to the death of these cells.

Course of the NETosis process. Cell death as a result of NETosis differs from necrosis and apoptosis. Shortly after induction of the process, protrusions occur in neutrophils and the cell flattened. There is decondensation of nuclear chromatin and its mixing with the granules of these cells. On the other hand, cell membrane folding, nuclear chromatin condensation, increased caspase activity and internucleosomal DNA cuts that occur in the process of apoptosis are not observed [21].

It should be noted that many mechanisms accompanying NETosis remain unexplained. However, it is believed that this process depends on three factors, i.e. histone citrullination, ROS release and autophagy [2, 21, 25, 26, 27, 28, 29].

It has been proved that histones are subject to many post-translational modifications, of which deimination (citrullination) of guanidine arginine residues in histones $\mathrm{H} 2 \mathrm{~A}, \mathrm{H} 3$ and $\mathrm{H} 4$ is important for NETosis [27]. There are five known peptidyl arginine deiminase (PAD) isoforms, which are capable of performing such deimination, of which PAD4 is the most frequently described form [28]. PAD4 catalyses the conversion of arginine residues to citrulline polypeptides, thereby it eliminates the positive charge of the protein. Thus, histone citrullination weakens the stability of nucleosomes $[29,30]$. Loss of positive charges results in the opening of the compact chromatin structure and enables decondensation of chromatin in the form of NET. Neutrophils of PAD4deficient mice show limited ability to form NETs and are very susceptible to severe skin infections in vivo [31,32].

To-date, researchers have not been able to discover how the activity and expression of PAD4 can regulate the functions of neutrophils, although certainly citrullination is a very important, but insufficient element modifying the course of NETosis. Probably, activation of additional mechanisms is necessary, thanks to which the formation of NET is possible [31]. ROS play a significant role in the release of chromatin into the extracellular space. The formation of ROS depends on NADPH oxidase, Raf-MEK_ERK and p38MAPK pathways. Properly stimulated neutrophils activate NADPH oxidase, contributing to the production of peroxides, while suppression of the NADPH oxidase complex, e.g. by diphenyleneiodonium (DPI), blocks the release of NET [21].
Generation of a PMA-induced NET is not possible in people who have a mutation of any of the four genes coding for the NADPH oxidase subunits. This condition occurs in patients with CDG (chronic granulomatous disease). However, after the incubation of neutrophils with glucose oxidase, hydrogen peroxide is produced, which is able to pass through the cell membrane and induce NET $[33,34]$. The mechanism of creating the NET is shown in Figure 1.

In studies conducted by Remijsen et al. [28] using a non-specific inhibitor of autophagy - wortmannin, it was demonstrated that the occurrence of NETosis in neutrophils stimulated with PMA requires a correct course of activation of NADPH oxidase, and the ability of neutrophils to perform the process of autophagy. Moreover, both processes are required to inhibit the activation of caspases and subsequent cell death by apoptosis. Total chromatin decondensation dependent on autophagy in intact neutrophils and inhibition of caspases may contribute to the emergence of functional NETs. Patients with CDG are not able to produce ROS. However, pharmacological inhibition of autophagy does not inhibit the oxidative burst dependent on NADPH oxidase, and the inhibition of NADPH oxidase has no effect on the autophagy process. However, disturbances in the course of any of these two mechanisms block the decondensation of nuclear chromatin, and thus contribute to abnormalities in NET formation [28].

\section{DIRECT METHODS OF NETOSIS PROCESS OBSERVATION}

Microscopy. Some of the methods of NETosis imaging are fluorescence electron microscopy and SEM (scanning electron microscopy). In this method, neutrophils are placed on glass plates with chambers, incubated with or without stimulation for several hours, fixed, and then subjected to immunostaining. The conventional reagents used, such as acetone and ethanol, pass through the plasmatic membrane at the time of cell fixing and thus they can induce artificial formation of NET. SEM uses a focused beam of electrons to obtain images with high resolution and large magnification formed by secondarily dispersed electrons emitted from the sample surface. Due to the focused excitation beam, the obtained images are characterised with a large depth of field that can be used to understand the molecule topography. Pérez-Sánchez C et al. [47] used fluorescence electron microscopy, staining neutrophils with DAPI (4区, 6-diamidino-2-phenylindole) stain and scanning electron microscopy in patients with RA (rheumatoid arthritis) and in healthy subjects. The neutrophils were fixed in $2.5 \%$ glutaraldehyde solution, washed and coated with gold using High Vacuum Coater Leica EM ACE600. This is a system used to prepare a sample and then to visualize it using a scanning electron microscope. Samples were viewed and photographed using a scanning microscope. In comparison to healthy people, neutrophils from RA patients showed significantly more spontaneous NET formation.

The above described process was evaluated by means of fluorescence microscopy and scanning electron microscopy. The higher percentage of cells undergoing NET in patients with RA was also associated with increased expression of $\mathrm{NE}$ and MPO. In order to evaluate the expression of $\mathrm{NE}$ and MPO proteins, the neutrophils were incubated with human anti-MPO antibody or with human primary anti-NE 
Table 1. NET elements important in the process of NETosis

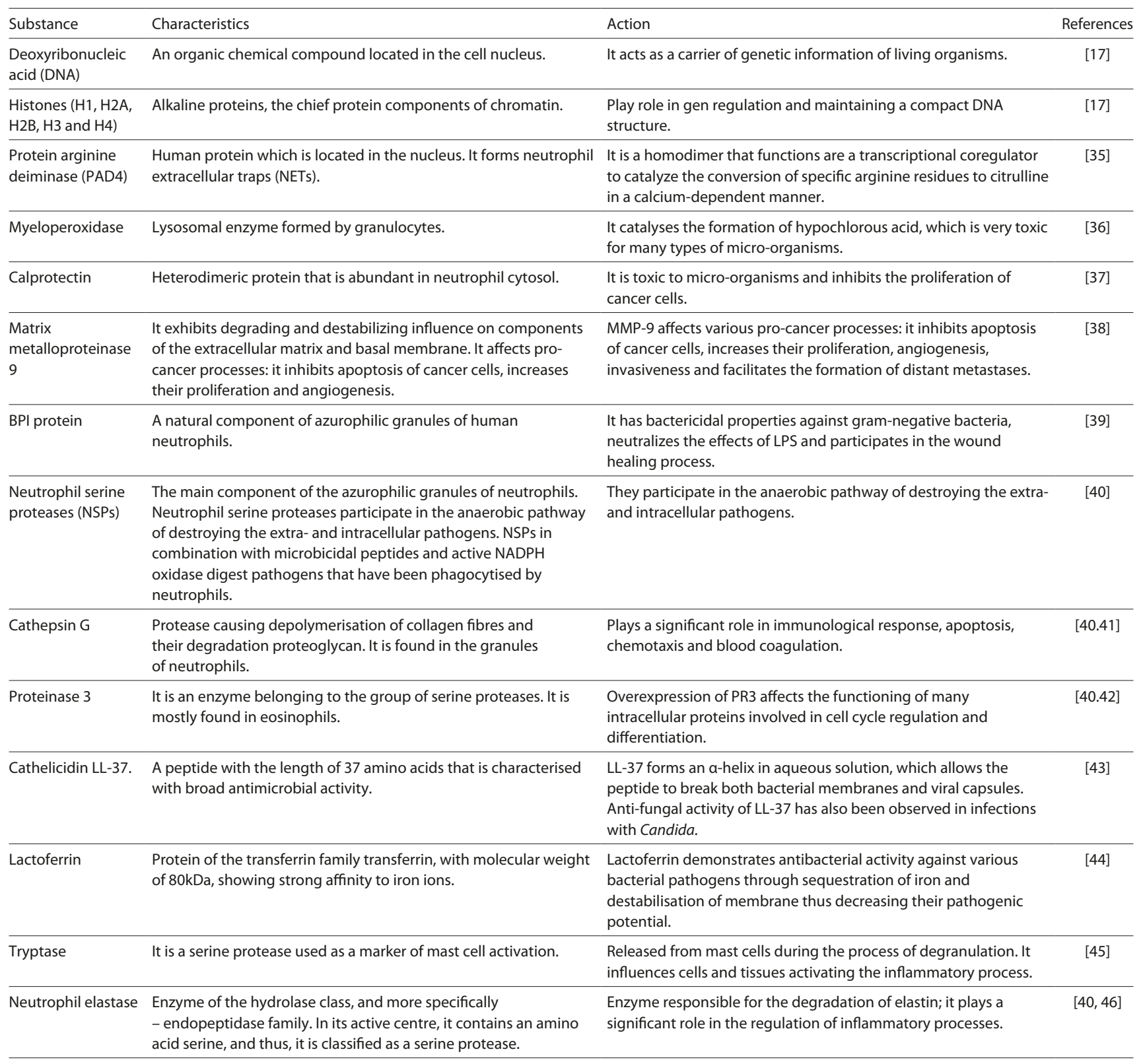

antibody and with secondary antibody. The cells were then rinsed and analysed using a flow cytometer - FACSCalibur [47]. Remijsen et al. observed in 2011 that morphological difference between NET and fibrin using an SEM are invisible and unclear. One of the disadvantages of using SEM to NETosis imaging is the uncertainty that the NETosis process takes place [48].

ThenextmethodforimagingNETosisisimmunofluorescence microscopy. Buhr et al. used immunofluorescence microscopy to evaluate the formation of NETs in CSF (cerebrospinal fluid). The study was conducted in piglets infected with Streptococcus suis. In this CSF, NET fibres were detected, but in a healthy control group, NET was not found [49].

Another method to illustrate NETosis entails using CLSM (confocal laser scanning microscopy). This is a modern variation of fluorescence microscopy, characterized however, by better resolution and contrast compared to ordinary fluorescence microscopes. It is a method of NETosis visualisation in -vitro by using a semi-automatic 3D CLSM image analysis. The image is obtained by scanning the surface of the preparation with a laser, which simultaneously stimulates the fluorescence of the dye. This method is characterized by increased sensitivity in detecting the emergence of NET when the process is not intensified. It is therefore a semi-automatic and relatively objective method [50].

Kraaij et al. [50] applied the automatic neutrophil imaging technique using 3-dimensional confocal laser scanning microscopy (3D-CLSM). In their study, samples of blood were obtained from patients with RA, SLE, and from healthy volunteers. The results showed low levels of NET release after stimulation with human immune complexes, as opposed to high NET levels after PMA stimulation. Serum samples from patients with RA and SLE demonstrate the ability to induce NET, compared to serum from healthy people. Several patients with RA or SLE showed increased ability to induce NETs.

In summary, microscopy is a specific NETosis imaging technique which has certain limitations to be observer- 
dependent and it is time-consuming. SEM might be used with immunostaining of certain structures in NETs. The other advantage of SEM is the visible differentiation between NETosis and necrosis. One of the disadvantages is that NETs might be confused with fibrin [51].

Flow cytometry and its modifications. The emergence of measurements using flow cytometry was an important step towards understanding individual traits within a given population. This technique allows for fast measurement of scattered light or fluorescence signals emitted by properly irradiated cells. It allows for both qualitative and quantitative evaluation of the physical and biological properties of cells and some of their components: nuclei, nucleic acids, mitochondria. The invention of flow cytometry constitutes an improvement of the fluorescence microscope and is characterized by high efficiency, which allows for analysing various parameters in a relatively short time $[52,53,54]$.

Gavillet et al. [55] described a flow cytometry test used for the identification and quantification of NET with antibodies against its key components, in particular DNA, modified histones and granule enzymes. This method has been applied to both mouse and human samples to evaluate the induced NETs in vitro - detect NETosis - in blood samples. The study used human blood collected on ethylenediaminetetraacetic acid (EDTA) suspended in RPMI1640. The samples were subjected to qualitative and quantitative analysis. Using a cytometer, NET was quantitatively determined directly in blood samples. Hence, the significant NET formation was demonstrated in samples stimulated with ionomycin, compared to unstimulated samples.

A microscope was also used to verify the NETosis process observed in the flow cytometer. No visible microscopic image changes were observed in the control group, cells showed typical nuclei with multiple shoots, while in PMN stimulated cells, visible changes were observed, neutrophils showed the extracellular fibrous DNA morphology of a 'shooting star'.

Next, a microscope and a flow cytometer were used to detect NET in blood samples collected on EDTA from healthy subjects and from patients with sepsis. In the microscopic image, the cells from patients showed DAPI+, MPO+, H3Cit+ (histone citrullination promotes chromatin decondensation and is considered a key marker of early NETosis), while neutrophils in healthy volunteers showed typical nuclei with multiple shoots (DAPI+, H3Cit-, MPO-). In both cases, the NETosis process was detected. However, in the control group, NETosis was less pronounced than in patients with sepsis.

All the samples from patients with sepsis showed significantly increased NET values. These data indicate that
NET can be directly quantified in blood samples by means of flow cytometry, and that this technique makes it significantly easier to analyse clinical blood samples, as it allows for quick assessment of a much larger number of cells in a given sample [46]. The advantage of using flow cytometry is the possibility of further sorting and differentiating between selected cell populations [51].

Masuda et al. [33] also performed a quantitative evaluation of NET detection using flow cytometry. They used a DNA binding dye that was unable to pass through the membrane - SYTOX Green. The green stained cells were detected in PMNs subjected to PMA. The number of green stained cells increased depending on the time of exposure and the concentration of PMA. The obtained data suggest that the green stained DNA came from the neutrophils that formed NET. Human PMN was isolated from the peripheral blood of healthy volunteers.

Another method to determine the NETosis process is the multispectral image flow cytometry (MIFC), combining the features of fluorescence microscopy and flow cytometry. This method uses and analyses a large number of images from suspended cells. In order to obtain the image, transmitted light (Brightfield), side-scattered light (SSC) and fluorescent images of cellular components are used [56]. In a study conducted by Zhao et al. [56], the researchers evaluated neutrophils for the morphology of their nucleus and the distribution of MPO and DNA in the cell. Peripheral blood samples were analysed in healthy volunteers and patients with SLE (systemic lupus erythematosus). Circulating neutrophils of normal density in SLE patients showed significantly more severe NETosis ex vivo, in the absence of an exogenous stimulus, compared to healthy control unstimulated neutrophils.

The fluorescence evaluation was then performed using the BDI (Bright Detail Intensity) function. Decondensation nuclei (cells with NETosis observed in patients with SLE) are identified by low BDI and a high fluorescence area, while normal nuclei show high or variable BDI and low fluorescence. This method allows for easy visualisation of the NETosis process in neutrophils. Overall, these results indicate that MIFC can be used as a quick method for comparing the percentage of neutrophils undergoing NETosis in various diseases [56].

One of the greatest advantages of this method is that it is automated and can be combined with sorting. Its disadvantage is that it does not detect events independent of H3cit [51]. This method is also limited due to the fact that it tests the cells currently undergoing NETosis, and may miss the cells that are in a late phase of NETosis [56].

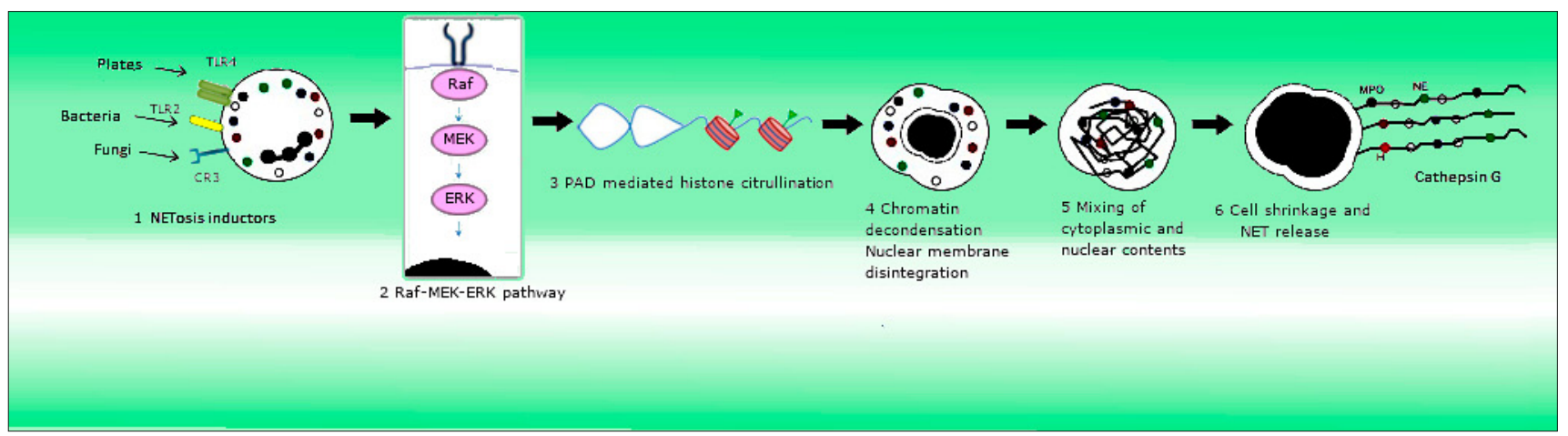

Figure 1. Mechanism of NETosis formation [34] 
Technique of NETosis real time imaging. Gupta et al. [57] developed and optimized a new method of automatic real time computing of the percentage of human neutrophils undergoing NETosis by means of the IncuCyte ZOOM imaging platform using the properties of cell membranes associated with permeability of DNA dyes (Sytox Green and NUCLEAR-ID). The IncuCyte ZOOM system is configured to quantify cells in three imaging channels: phase contrast channel, green fluorescence and red fluorescence. The use of images with phase contrast allows the evaluation the cell morphology, the red NUCLEAR-ID dye, which freely crosses the cell membrane, binds to nucleic acids and lights up red, as well as stains and counts cell nuclei, whereas the green SYTOX Green dye stains dead cells. Moreover, by using high-resolution phase contrast imaging in conjunction with a fluorescence dye signal, the IncuCyte ZOOM platform was able to distinguish between different types of neutrophil death induced by various stimuli based on morphological characteristics of the cell. Due to the fact that the system is functioning in a controlled cell environment, the platform provides insight into active biological processes occurring in real time, which is impossible using the single point and final measurements.

Neutrophils undergoing NETosis induced by various physiological stimuli, i.e. nigericin $(0.5 \mathrm{mM})$, calcium ionophore $(25 \mathrm{mM})$ and PMA $(0.5 \mathrm{mM})$, showed distinctive changes, e.g. chromatin decondensation. These cells were accurately counted using filters based on the evaluation of the intensity, radius and fluorescence area as well as the size of the nucleus. The findings were confirmed using immunofluorescence microscopy. The platform has also been validated in order to quickly evaluate and quantify the dose-dependent effect of NETosis inhibitors. Additionally, this method was able to distinguish neutrophils undergoing NETosis, apoptosis or necrosis based on clear changes in nuclear morphology and membrane integrity. The IncuCyte ZOOM platform is an innovative real-time test that measures NETosis in a fast, automatic and reproducible manner and significantly optimizes neutrophil testing [57].

Lovell et al. [58] using cell-staining reagent (IncuCyte CytoTox Green) visualized and quantified the release of NET in real-time. The addition of the IncuCyte reagent to healthy cells does not interfere with cell growth and morphology and produces a small or negligible internal fluorescence signal. The reduced cell membrane integrity allows the IncuCyte cytotoxic reagent to enter the cell and cause a 100-1,000fold increase in fluorescence after binding to DNA. Both in human neutrophils and dHL60-differentiated neutrophillike cells, induced with PMA (100 nM), a time-dependent increase in fluorescence and nuclear degradation was demonstrated. Simultaneous increase in MPO, NE and nonnuclear DNA was observed, confirming the NETosis signal.

The main advantage of the IncuCyte technique is the possibility of direct evaluation of cell morphology and differentiation of the mechanisms of cell death induced by various stimuli. That method quantifies the percentage of neutrophils undergoing the process of NETosis, and does not quantify extracellular DNA [57].

Gupta et al. used a fluorometer to quantified NET formation. This technique requires the use of a DNA dye excluded from cells. It was applied to detect extracellular DNA release from neutrophils. After stimulating human cells with PMA, IL-8, ionomycin measures fluorescence of cells. The fluorescence level of the treated cells was obtained after subtracting the baseline fluorescence of unstimulated cells [59]. Pieterse et al. used the colorimetrical method for evaluation neutrophil elastase and myeloperoxidase. Depending on the inductor used, the amount of granular enzyme activities was different [60]. One of the disadvantages of the above method is that the occasional staining of DNA in NETs can be blocked by cationic peptides [51].

In-vivo and in-situ methods. Many scientists have discovered new methods for visualizing the NETs, and several research groups have used intravital microscopy for in-vivo method of imaging NETosis, based on DNA components [51]. In 2007 Clark et al. developed data about releasing NETs in response to platelet activation by neutrophils, using intravital microscopy of the liver sinusoid [18]. Subsequent research demonstrated the NET formation in the carotid bifurcation using intravital two-photon microscopy, in the liver sinusoids using spinning disc confocal intravital microscopy, and intravenously using two-photon with epifluorescence microscopy. The majority of in-vivo experiments were performed on murine species by intravital microscopy [51].

The in-situ method might be used for visualisation NETosis in human and animal cells. A special case for in-situ NET analysis is the live-cell visualisation performed immediately after euthanasia of the animals. This process is carried out without fixation of the selected organs. NET was detected in-situ in lungs, infected with Aspergillus fumigatus, using two-photon microscopy. The lungs were prepared, dissected, and visualized with SYTOX dye. During the analysis the lung was stored in PBS at $37^{\circ} \mathrm{C}$ [51].

The majority of in-vivo and in-situ experiments detected fewer NET structures compared to the results from invitro experiments. This effect may be made by a complex time-dependent regulatory process of NET induction and elimination within the host. Different live-cell imaging methods with in-vivo experiments have been widely used and considered to be an excellent method to exemplify NET release in the case of an autoimmune disease or infection [51].

\section{INDIRECT METHODS OF NETOSIS PROCESS OBSERVATION}

Immunostaining (ELISA). Another technique for determining the NETosis process markers is ELISA (enzyme-linked immunosorbent assay). This technique allows for, among others, the evaluation of MPO-DNA and NE--DNA complexes in liquid samples. ELISA test plates are covered with anti-MPO antibody. Then, horseradish peroxidase labelled anti-DNA antibodies are used. There is a change in the colour of the substrate in which the reaction takes place. The increased release of $\mathrm{H} 3 \mathrm{Cit}$ by neutrophils in vitro and the positive correlation between the MPODNA complexes indicate NETosis process taking place in neutrophils. Some studies have shown an increase in analyzed NETs markers, and an elevated level of MPO-DNA complex in the serum of patients with controlled type 2 diabetes mellitus and vasculitis associated with the presence of ANCA (anti-neutrophil cytoplasmic antibodies) [61].

Thålin et al. [61] developed a new method for the determination of H3Cit by means of ELISA which can be used to detect NETosis in human plasma. The results 
confirmed linearity and high specificity of $\mathrm{H} 3 \mathrm{Cit}$, obtained by the inability to detect non-citrullinated $\mathrm{H} 3$ histone. Intraand inter-assay variability ratios indicates high accuracy of determinations. The results also confirmed a marked increase in H3Cit concentration in plasma in the human LPS-induced inflammation model [61].

In a study by Thålin et al. [62] ELISA was used to assess proteins in patients with and without cancer. In patients with advanced cancer, analysis showed a significantly higher H3Cit compared to chronically ill patients (i.e. patients without cancer, but with other diseases, e.g. hypertension, congestive heart disease, type I or II diabetes, dementia, renal and hepatic failure, chronic lung disease and acute infections). It has been demonstrated that the presence of $\mathrm{H} 3 \mathrm{Cit}$ in cancer patients strongly correlates with markers of neutrophil activation such as: NE and MPO, and NETosis inducing inflammatory cytokines IL-6 and IL-8. Moreover, a high concentration of $\mathrm{H} 3 \mathrm{Cit}$ in plasma translated into a worse prognosis in the course of disease treatment. Plasma samples were prepared from whole blood collected on citrate. Quantification of H3Cit was obtained using streptavidincoated plates, biotinylated antihistone antibody as capturing antibody, and anti-histone $\mathrm{H} 3$ antibody citrulline as detecting antibody. MPO-DNA complexes were also identified by means of ELISA. The MPO-DNA complexes were bound to microplates coated with anti-MPO monoclonal antibodies and then marked with anti-DNA antibodies. Cancer patients showed a positive correlation between $\mathrm{H} 3 \mathrm{Ci}$ and circulating free DNA (cfDNA) levels, neutrophil activation markers and MPO-DNA complexes, suggesting that the presence of circulating H3Cit may be associated with neutrophil and NET activation. Patients with chronic disease not diagnosed with cancer showed similar correlations between cfDNA, neutrophil activation markers and MPO-DNA complexes, with correlations between $\mathrm{H} 3 \mathrm{Cit}$ and neutrophil activation markers being weaker or close to zero. Increased neutrophil activity did not significantly increase the formation of NET in these patients.

To sum up, the results suggest a similar level of neutrophil activation in cancer patients and non-cancer patients, although there is an increased release of $\mathrm{H} 3 \mathrm{Cit}$ by neutrophils in cancer patients due to the NETosis process [62]. Margraf et al. examined the level of free DNA which is used as a marker of NETs in serum samples, usually detected by a fluorescent quantitative study. The PicoGreen dye is most commonly used in research, which binds to double stranded cfDNA. Circulating free DNA/NETs seems to be a valuable proinflammatory marker [63].

\section{CONCLUSIONS}

After the discovery of NET in 2004, Brinkmann et al. conducted numerous studies on their role and the possibility of assessing this process in many diseases. Despite the development of the technique, there are still no methods to assess and determine NETosis in an objective, reproducible and effective manner, which significantly limits the progress of research in this field. Researchers should choose the most appropriate marker or method in each situation based on their knowledge of their respective advantages and disadvantages. The disadvantage of the most commonly used methods, i.e. microscopy, is the lack of objectivity and the difficulty in quantitative evaluation of this process. Contrary to microscopy, flow cytometry allows for specific, objective and quantitative determination of NET in blood samples (many cells may be assessed simultaneously). However, this method is also burdened with several disadvantages, including: high cost of the equipment required to conduct research, and the need to have experience in operating a cytometer (complicated service and frequent difficulties with unambiguous interpretation of the observed results). As an alternative to expensive flow cytometry methods, there are sensitive, highly specific and cheaper immunoenzymatic ELISA tests. Although the problem with standardisation of ELISA test results remains unresolved, this technique seems to be the most current, objective and quantitative NETosis monitoring method.

\section{REFERENCES}

1. Martin SJ, Henry CM. Distinguishing between apoptosis, necrosis, necroptosis and other cell death modalities. Methods, 2013; 161(2): 87-9.

2. Brinkmann V, Reichard U, Goosmann C, Fauler B, Uhlemann Y, Weiss D.S, Weinrauch Y, Zychlinsky A. Neutrophil Extracellular Traps Kill Bacteria. Science, 2004; 303: 1532-1535.

3. Homa-Mlak I, Majdan A, Mlak R, Małecka-Masalska T. Metastatic potential of NET in neoplastic disease. Postępy Hig. Med. Dośw. 2016; 70: 887.-895.

4. Matoszka N, Działo J, Tokarz-Deptuła B, Deptuła W. NET and NETosis - new phenomenon in immunology. Postępy Hig. Med. Dośw. 2012; 66: 437-445.

5. Beiter K, Wartha F, Albiger B, Normark S, Zychlinsky A, Normark H.B.: An endonuclease allows Streptococcus pneumoniae to escape from neutrophil extracellular traps. Curr. Biol. 2006; 16: 401-407.

6. Buchanan J.T, Simpson A.J, Aziz R.K, Liu G.Y, Kristian S.A, Kotb M, Feramisco J, Nizet V. DNase expression allows the pathogen group A Streptococcus to escape killing in neutrophil extracellular traps. Curr. Biol. 2006; 16: 396-340.

7. Ramos-Kichik V, Mondragón-Flores R, Mondragón-Castelán M, Gonzales-Pozos S, Muniz-Hernandez S, Rojas-Espinosa O, ChacónSalinas R, Estrada-Parra S, Estrada-Garcia I. Neutrophil extracellular traps are induced by Mycobacterium tuberculosis. Tuberculosis, 2009; 89: 29-37.

8. Bianchi M, Niemiec M.J, Siler U, Urban C.F, Reichenbach J. Restoration of anti-Aspergillus defense by neutrophil extracellular traps in human chronic granulomatous disease after gene therapy is calprotectindependent. J. Allergy Clin. Immunol. 2011; 127: 1243-1252.e7.

9. Urban C.F., Ermert D., Schmid M., Abu-Abed U., Goosmann C., Nacken W., Brinkmann V., Jungblut P.R, Zychlinsky A. Neutrophil extracellular traps contain calprotectin, a cytosolic protein complex involved in host defense against Candida albicans. PLoS Pathog. 2009; 5: e1000639.

10. Urban C.F, Reichard U, Brinkmann V, Zychlinsky A. Neutrophil extracellular traps capture and kill Candida albicans yeast and hyphal forms. Cell. Microbiol. 2006; 8: 668-676.

11. Gabriel C, McMaster W.R, Girard D, Descoteaux A. Leishmania donovani promastigotes evade the antimicrobial activity of neutrophil extracellular traps. J. Immunol. 2010; 185: 4319-4327.

12. Guimarães-Costa A.B, Nascimento M.T, Froment G.S, Soares R.P, Morgado F.N, Conceição-Silva F, Saraiva E.M. Leishmania amazonensis promastigotes induce and are killed by neutrophil extracellular traps. Proc. Natl. Acad. Sci. USA, 2009; 106: 6748-6753.

13. Papayannopoulos V, Zychlinsky A. NETs: a new strategy for using old weapons. Trends Immunol. 2009; 30: 513-52.

14. Carmona-Rivera C, Kaplan M.J. Low density granulocytes: a distinct class of neutrophils in systemic autoimmunity. Semin Immunopathol. 2013; 35(4): 455-463.

15. Gupta A.K, Joshi M.B, Philippova M, Erne P, Hasler P, Hahn S, Resink T.J. Activated endothelial cells induce neutrophil extracellu- lar traps and are susceptible to NETosis-mediated cell death. FEBS Lett. 2010; 584: 3193-3197.

16. Lande R, Ganguly D, Facchinetti V, Frasca L, Conrad C, Gregorio J, Meller S, Chamilos G, Sebasigari R, Riccieri V, Bassett R, Amuro H, Fukuhara S, Ito T, Liu Y.J, Gilliet M.: Neutrophils activate pla- smacytoid dendritic cells by releasing self-DNA-peptide complexes in systemic lupus erythematosus. Sci. Transl. Med., 2011; 3: 73ra19. 
17. Yang H, Biermann M, Brauner J, Liu Y, Zhao Y, Herrmann M. New Insights into Neutrophil Extracellular Traps: Mechanisms of Formation and Role in Inflammation. Front Immunol. 2016; 7: 302.

18. Clark SR, Ma AC, Tavener SA, McDonald B, Goodarzi Z, Kelly MM, Patel KD, Chakrabarti S, McAvoy E, Sinclair GD, Keys EM, Allen-Vercoe E, Devinney R, Doig CJ, Green FH, Kubes P. Platelet TLR4 activates neutrophil extracellular traps to ensnare bacteria in septic blood. Nat. Med., 2007; 13: 463-469.

19. Ma A.C, Kubes P. Platelets, neutrophils, and neutrophil extracellu- lar traps (NETs) in sepsis. J. Thromb. Haemost. 2008; 6: 415-420.

20. Guichard C, Pedruzzi E, Dewas C, Fay M, Pouzet C, Bens M, Vandewalle A, Ogier-Denis E, Gougerot-Pocidalo M.A, Elbim C. Interleukin-8induced priming of neutrophil oxidative burst requires sequential recruitment of NADPH oxidase components into lipid ra- fts. J. Biol. Chem. 2005; 280: 37021-37032.

21. Fuchs T.A, Abed U, Goosmann C, Hurwitz R, Schulze I, Wahn V Weinrauch Y, Brinkmann V, Zychlinsky A. Novel cell death program leads to neutrophil extracellular traps. J. Cell Biol, 2007; 176: 231-24.

22. Yousefi S, Gold J.A, Andina N, Lee J.J, Kelly A.M, Kozlowski E, Schmid I, Straumann A, Reichenbach J, Gleich G.J, Simon H.U: Catapultlike release of mitochondrial DNA by eosinophils contribu- tes to antibacterial defense. Nat. Med 2008; 14: 949-953.

23. Simon D, Hoesli S, Roth N, Staedler S, Yousefi S, Simon H.U. Eosinophil extracellular traps in skin diseases. J. Allergy Clin. Immunol 2011; 127: 194-199.

24. Chow O.A, von Köckritz-Blickwede M, Bright A.T, Hensler M.E, Zinkernagel A.S, Cogen A.L, Gallo R.L, Monestier M., Wang Y, Glass C.K, Nizet V. Statins enhance formation of phagocyte extra- cellular traps. Cell Host Microbe 2010; 8: 445-454.

25. Masuda S, Nakazawa D, Shida H, Miyoshi A, Kusunoki Y, Tomaru U, IshizuA. NETosis markers: Quest for specific, objective, and quantitative markers. Clinica Chimica Acta 2016; 459; 89-93.

26. Neeli I, Dwivedi N, Khan S, Radic M. Regulation of extracellular chromatin release from neutrophils. J. Innate Immun 2009; 1: 194-201.

27. Neeli I, Khan S.N, Radic M. Histone deimination as a response to inflammatory stimuli in neutrophils. J. Immunol 2008; 180: 1895-1902.

28. Remijsen Q, Berghe T.W, Wirawan E, Asselbergh B, Parthoens E, de Rycke R, Noppen S, Delforge M, Willems J, Vandenabeele P: Neutrophil extracellular trap cell death requires both autophagy and superoxide generation. Cell Research 2011; 21: 290-304.

29. Wang Y, Li M, Sonja S, Sarah C, Pingxin L, Wang DC, et al. Histone hypercitrullination mediates chromatin decondensation and neutrophil extracellular trap formation. J Cell Biol 2009;184(2): 205-13.

30. Wang Y, Wysocka J, Sayegh J, Lee YH, Perlin JR, Leonelli L. Human PAD4 regulates histone arginine methylation levels via demethylimination. Science, 2004; 306(5694): 279-83.

31. Pingxin L, Ming L, Lindberg MR, Kennett MJ, Na X, Yanming W. PAD4 is essential for antibacterial innate immunity mediated by neutrophil extracellular traps. J Exp Med 2010; 207(9): 1853-62.

32. Saskia H, John RT, Sanja A, Kerra AM. PAD4-mediated neutrophil extracellular trap formation is not required for immunity against influenza infection. PLoS One, 2011; 6(7): e22043.

33. Masuda S, Shimizu S, Matsuo J, Nishibata Y, Kusunoki Y, Hattanda $\mathrm{F}$, et al. Measurement of NET formation in vitro and in vivo by flow cytometry. Cytometry A 2017; 91(8): 822-829.

34. Papayannopoulos V. Neutrophil extracellular traps in immunity and disease. Nat Rev Immunol 2018;18(2):134-147.

35. Jones J, Causey C, Knuckley B, Slack-Noyes J. L, Thompson P. R. Protein arginine deiminase 4 (PAD4): current understanding and future therapeutic potential Curr Opin Drug Discov Devel 2009; 12(5): 616-627.

36. Droeser R.A, Hirt C, Eppenberger-Castori S, Zlobec I, Viehl C.T, Frey D.M, et al. High myeloperoxidase positive cell infiltration in colorectal cancer is an independent favorable prognostic factor. PLoS One 2013; 8: e64814.

37. Srikrishna G. S100A8 and S100A9: new insights into their roles in malignancy. J. Innate Immun., 2012; 4: 31-40.

38. Tazzyman S, Niaz H, Murdoch C. Neutrophil-mediated tumour angiogenesis: subversion of immune responses to promote tumour growth. Semin. Cancer Biol 2013; 23: 149-158.

39. van der Schaft D.W, Wagstaff J, Mayo K.H, Griffioen A.W. The antiangiogenic properties of bactericidal/permeability-increasing protein (BPI). Ann. Med 2002; 34: 19-27.

40. Korkmaz B, Horwitz M.S, Jenne D.E, Gauthier F. Neutrophil elastase, proteinase 3, and cathepsin $\mathrm{G}$ as therapeutic targets in human diseases. Pharmacol. Rev 2010; 62: 726-759.

41. Mayadas T.N, Cullere X, Lowell C.A. The multifaceted functions of neutrophils. Annu. Rev. Pathol 2014; 9: 181-218.

42. Witko-Sarsat V, Canteloup S, Durant S, Desdouets C, Chabernaud R, Lemarchand P, Descamps-Latscha B.: Cleavage of p21waf1 by proteinase-3, a myeloid-specific serine protease, potentiates cell proliferation. J. Biol. Chem 2002; 277: 47338-47347.

43. Reinholz M, Ruzicka T, Schauber J. Cathelicidin LL-37: An Antimicrobial Peptide with a Role in Inflammatory Skin Disease. Ann Dermatol 2012; 24(2): 126-135.

44. Jenssen H, Hancock RE. Antimicrobial properties of lactoferrin. „Biochimie” 2009; 91 (1): 19-29.

45. Tanaka T, McRae BJ, Cho K, Cook R, Fraki JE, Johnson DA, Powers JC. (November 1983). Mammalian tissue trypsin-like enzymes. Comparative reactivities of human skin tryptase, human lung tryptase, and bovine trypsin with peptide 4-nitroanilide and thioester substrates The Journal of Biological Chemistry.1983; 258 (22): 13552-7.

46. Gong L, Cumpian A.M, Caetano M.S, Ochoa C.E, De la Garza M.M., Lapid D.J, Mirabolfathinejad S.G, Dickey B.F, Zhou Q, Moghaddam S.J. Promoting effect of neutrophils on lung tumorigenesis is mediated by CXCR2 and neutrophil elastase. Mol. Cancer, 2013; 12: 154.

47. Pérez-Sánchez C, Ruiz-Limón P, Aguirre MA, Jiménez-Gómez Y,Ariasde la Rosa I,Ábalos-Aguilera MC, et al. Diagnostic potential of NETosisderived products for disease activity, atherosclerosis and therapeutic effectiveness in Rheumatoid Arthritis patients. J Autoimmun, 2017; 82: $31-40$.

48. Remijsen Q, Kuijpers T. W, Wirawan E, Lippens S, Vandenabeele P, Vanden Berghe T. Dying for a cause: NETosis, mechanisms behind an antimicrobial cell death modality. Cell Death Differ 2011;18(4):581-8.

49. De Buhr N, Reuner F, Neumann A, Stump-Guthier C, Tenenbaum T, Schroten H. et al.: Neutrophil extracellular trap formation in the Streptococcus suis-infected cerebrospinal fluid compartment. Cell Microbiol 2017;19(2).

50. Kraaij T, Tengström FC, Kamerling SW, Pusey CD, Scherer HU,Toes RE, Rabelink TJ, van Kooten C.,Teng YK. A novel method for highthroughput detection and quantification of neutrophil extracellular traps reveals ROS-independent NET release with immune complexes. Autoimmun Rev 2016; 15(6): 577-84.

51. De Buhr N, von Köckritz-Blickwede M. How Neutrophil Extracellular Traps Become Visible. Journal of Immunology Research 2016, Article ID 4604713, 13 pages.

52. Alvarez D. F, Helm K, DeGregori J, Roederer M, Majka S. Publishing flow cytometry data. American Journal of Physiology - Lung Cellular and Molecular Physiology 2010; 298(2): 127-130.

53. Ibrahim SF, van den Engh G. Flow cytometry and cell sorting. Adv Biochem Eng Biotechnol. 2007; 106: 19-39.

54. Skotny A, Pucińska J. Współczesna Cytometria przepływowa. Acta BioOptica et Informatica Medica. Inżynieria Biomedyczna 2013; (19)1: 3-11.

55. Gavillet M, Martinod K, Renella R, Harris Ch, Shapiro N.I, Wagner D.D, Williams D.A.: Flow cytometric assay for direct quantification of Neutrophil Extracellular Traps in blood sample. Am J Hematol, 2015; 90(12): 1155-1158.

56.Zhao W, Fogg D.K, Kaplan M.J: A novel image-based quantitative method for the characterization of NETosis. J Immunol Methods 2015; 423: $104-110$.

57. Gupta S,Chan DW, Zaal KJ, Kaplan MJ. A High-Throughput Real-Time Imaging Technique To Quantify NETosis and Distinguish Mechanisms of Cell Death in Human Neutrophils. J Immunol.2018; 200: 869-879.

58. Lovell G.F, Bevan N, Dale T, Trezise D. J. Real-time visualisation and quantification of Neutrophil Extracellular Traps. J Immunol, 2018: 200; (1)49.5.

59. Gupta A.K, Giaglis S, Hasler P, Hahn S. Efficient neutrophil extracellular trap induction requires mobilization of both intracellular and extracellular calcium pools and is modulated by cyclosporine A. 2014;12;9 (5):e97088. doi: 10.1371/journal.pone.0097088. eCollection 2014.

60. Pieterse E, Rother N, Yanginlar C, Hilbrands L. B, van der Vlag J. Neutrophils Discriminate between Lipopolysaccharides of Different Bacterial Sources and Selectively Release Neutrophil Extracellular Traps. Front Immunol 2016; 7: 484. Published online 2016 Nov 4. doi: 10.3389/fimmu.2016.00484.

61. Thålin Ch, Daleskog M, Göransson S.P, Schatzberg D, Lasselin J, Laska A.CH, et al. Validation of an enzyme-linked immunosorbent assay for the quantification of citrullinated histone $\mathrm{H} 3$ as a marker for neutrophil extracellular traps in human plasma. Immunol Res 2017; 65(3): 706-712.

62. Thålin $\mathrm{C}$, Lundström $\mathrm{S}$, Seignez $\mathrm{C}$, Daleskog $\mathrm{M}$, Lundström $\mathrm{A}$, Henriksson $\mathrm{P}$, et al. Citrullinated histone $\mathrm{H} 3$ as a novel prognostic blood marker in patients with advanced cancer. PLoS One 2018: 11; 13(1).

63. Margraf S, Lögters T, Reipen J, Altrichter J, Scholz M, Windolf J. Neutrophil-derived circulating free DNA (cf-DNA/NETs): a potential prognostic marker for posttraumatic development of inflammatory second hit and sepsis. Shock 2008; 30(4):352-8. doi: 10.1097/ SHK.0b013e31816a6bb1. 